\title{
Extended non-linear relations of elastic shells undergoing phase transitions
}

\author{
Wojciech Pietraszkiewicz ${ }^{1, *}$, Victor Eremeyev ${ }^{2}$, and Violetta Konopińska ${ }^{1}$ \\ ${ }^{1}$ Institute of Fluid-Flow Machinery, Polish Academy of Sciences, ul. Gen. J. Fiszera 14, 80-952 Gdańsk, Poland \\ ${ }^{2}$ South Scientific Center of RASci and Rostov State University, Zorge str. 5, 344090 Rostov on Don, Russia
}

\begin{abstract}
Dedicated to the memory of Professor Pavel Zhilin
The non-linear theory of elastic shells undergoing phase transitions was proposed by two first anthors in J. Elast. 79,67-86 (2004). In the present paper the theory is extended by taking into account also the elastic strain energy density of the curvilinear phase interface as well as the resultant forces and couples acting along the interface surface curve itself. All shell relations are found from the variational principle of stationary total potential energy. In particular, we derive the extended natural continuity conditions at coherent and/or incoherent surface curves modelling the phase interface. The continuity conditions allow one to establish the final position of the interface surface curve after the phase transition. The results are illustrated by an example of a phase transition in an infinite plate with a central hole.
\end{abstract}

\section{Introduction}

The non-linear mechanical theory of elastic shells with an account of occurence of the martensitic type phase transition (PT) in the material was developed by Eremeyev and Pietraszkiewicz [1]. The formulation was based on the dynamically and kinematically exact shell model [2-4] with displacements composed of work-averaged translations and rotations of the shell cross sections. Such a general shell model had a structure of the classical Cosserat surface [5] to which Professor Pavel Zhilin contributed in [6-8]. In particular, in [1] we derived new static natural continuity conditions at the curvilinear phase interface. To model the phase equilibrium we used in [1] the variational approach based on the Gibbs variational principle [9]. Variational methods in the theory of phase transitions were developed in many papers and books, see for example [10-12].

Within 3D theories of phase transformation phenomena the phase interface is usually modelled by a sharp smoothly evolving surface separating different material phases in the thermodynamic equlibrium state. The surface interface itself may be endowed with various additional fields modelling different types of PTs, see for example Grinfeld [10] and Podstrigach and Povstenko [13], where many references are given. In particular, Povstenko [14] reviewed various models of the surface interfaces and three-phase line interface junctions in 3D continuum and proposed to treat them as 2D and 1D continua of the Cosserat type, respectively. Within 2D theories of thin films and biomembranes undergoing PTs simple 1D models of the interface surface curve were discussed by Wang [15], Atai and Steigmann [16], Boulbitch [17], Bhattacharya and James [18], Shu [19], James and Rizzoni [20], Roytburd and Slutsker [21], Gurtin and Jabbour [22], and Rubin et al. [23]. However, neither of those 1D models is complete enough to be applied with the non-linear theory of shells with PTs developed in [1]. The model of PTs in martensitic films developed in [18-20] was based on the Cosserat-type membrane theory which is a special case of the theory of shells. In [1] we proposed the most general model of such thin-walled bodies undergoing PTs. Our formulation of shell theory is based on the statically and kinematically exact through-the-thickness reduction of three-dimensional description of the phenomenon to two-dimensional form written on the shell base surface [1-4]. Using the general theory of shells we can describe the stress-induced PTs taking into account not only the tangentional stiffness but also the bending as well as transverse shear and normal stiffnesses of such martensitic films.

The aim of this paper is to extend the results of [1] by taking into account also the elastic strain energy density of the phase interface itself as well as additional resultant forces and couples acting along the interface surface curve and at its intersections with the shell boundary contour. The curvilinear density allows one to model within shell theory also

\footnotetext{
* Corresponding author, E-mail: pietrasz@imp.gda.pl, Phone: +00485869 95263, Fax: +0048583416144
} 
generalized capillary type phenomena widely discussed in continuum mechanics with PT, $[10,13]$. The additional forces and couples along the curvilinear interface may appear as a result of exact reduction to 1D problem of the 3D PT phenomenon in a thin tube about the interface surface curve, as it is given for branching and self-intersecting shells in $[24,25]$.

After reviewing the kinematic relations in Sect. 2, the equilibrium problem of PTs in elastic shells is formulated in Sect. 3 in the weak form as the stationary problem for the functional of total potential energy (7). Stationarity conditions of the functional following from (11) give the local equilibrium equations and static natural boundary conditions known from [1-4]. Along the curvilinear interface we obtain the new, local, natural continuity conditions (13), (14) which extend those proposed in [1]. The latter conditions allow one to establish position of the interface curve in the thermodynamic equilibrium state of PT in shells. Coherent curvilinear interfaces and interfaces incoherent in rotations are discussed in more detail in Subsections 4.1 and 4.2, respectively, where specific results appropriate to those two cases are given. The extended theory of PTs in shells is illustrated by a simple example of an infinite plate with a central hole discussed in [1], but now endowed with an additional capillary tension along the interface circle.

\section{Kinematic relations}

Within the dynamically and kinematically exact theory of regular shells [2-4], a shell is a three-dimensional (3D) solid body identified in a reference (undeformed) placement $\kappa$ with a region $B$ of the physical space $\mathcal{E}$ having the 3D vector space $E$ as its translation space. Geometry of $B$ is described in the normal coordinates $\left(\theta^{\alpha}, \xi\right), \alpha=1,2$, where $\xi=0$ defines the regular base surface $M \subset B$, and $\xi \in\left[-h^{-}, h^{+}\right]$is the distance from $M$, with $h=h^{-}+h^{+}$the shell thickness. If $\boldsymbol{x} \in E$ is the position vector of $x \in M$, then the surface covariant and contravariant base vectors $\boldsymbol{a}_{\alpha}$ and $\boldsymbol{a}^{\beta}$ are defined by

$$
\boldsymbol{a}_{\alpha}=\frac{\partial \boldsymbol{x}}{\partial \theta^{\alpha}} \equiv \boldsymbol{x}, \alpha, \quad \boldsymbol{a}^{\beta} \cdot \boldsymbol{a}_{\alpha}=\delta_{\alpha}^{\beta}, \quad \boldsymbol{\eta}=\frac{1}{2} \epsilon^{\alpha \beta} \boldsymbol{a}_{\alpha} \times \boldsymbol{a}_{\beta},
$$

where $\boldsymbol{\eta}$ is the unit normal orienting $M$ and $\epsilon^{\alpha \beta}$ are contravariant components of the surface permutation tensor $\boldsymbol{\epsilon}$.

In the deformed placement $\gamma$ the shell can be represented by the position vector $\boldsymbol{y}=\chi(\boldsymbol{x}) \in E$ of the material base surface $N=\chi(M)$ with attached three directors $\boldsymbol{d}_{\alpha}, \boldsymbol{d}$ such that

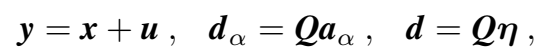

where $\chi$ is the deformation function, while $\boldsymbol{u} \in E$ is the translation vector of $M$ and $\boldsymbol{Q} \in S O(3)$ the proper orthogonal (rotation) tensor, $\boldsymbol{Q}^{T}=\boldsymbol{Q}^{-1}$, $\operatorname{det} \boldsymbol{Q}=+1$, describing the work-averaged gross deformation of the shell cross section.

The shell strain and bending tensors $\boldsymbol{E}$ and $\boldsymbol{K}$ in the spatial representation can be defined by [1-3]

$$
\begin{aligned}
& \boldsymbol{E}=\boldsymbol{\varepsilon}_{\alpha} \otimes \boldsymbol{a}^{\alpha}, \quad \boldsymbol{K}=\boldsymbol{\kappa}_{\alpha} \otimes \boldsymbol{a}^{\alpha}, \\
& \boldsymbol{\varepsilon}_{\alpha}=\boldsymbol{y},_{\alpha}-\boldsymbol{d}_{\alpha}=\boldsymbol{u},_{\alpha}+(\mathbf{1}-\boldsymbol{Q}) \boldsymbol{a}_{\alpha}=E_{\alpha \beta} \boldsymbol{d}^{\beta}+E_{\alpha} \boldsymbol{d}, \\
& \boldsymbol{\kappa}_{\alpha}=a x\left(\boldsymbol{Q},{ }_{\alpha} \boldsymbol{Q}^{T}\right)=\frac{1}{2}\left\{\boldsymbol{d}^{\beta} \times\left(\boldsymbol{Q},{ }_{\alpha} \boldsymbol{Q}^{T}\right) \boldsymbol{d}_{\beta}+\boldsymbol{d} \times\left(\boldsymbol{Q},{ }_{\alpha} \boldsymbol{Q}^{T}\right) \boldsymbol{d}\right\}=\boldsymbol{d} \times K_{\alpha \beta} \boldsymbol{d}^{\beta}+K_{\alpha} \boldsymbol{d} .
\end{aligned}
$$

In a two-phase elastic shell different material phases may appear in different complementary subregions $N_{A}$ and $N_{B}$ of $N$ separated by the curvilinear phase interface $D \in N$, Fig.1. For a continuous deformation $\chi$ we can introduce on $M$ a singular image curve $C=\chi^{-1}(D)$ separating the corresponding image regions $M_{A}=\chi^{-1}\left(N_{A}\right)$ and $M_{B}=\chi^{-1}\left(N_{B}\right)$. The position vectors of $C$ and $D$ are related by $\boldsymbol{x}_{C}(s)=\chi^{-1}\left(\boldsymbol{y}_{C}(s)\right)$, where $s$ is the arc length parameter along $C$.

The equilibrium boundary value problem (BVP) for shells with PTs was formulated in [1] in the weak form as the stationary problem for the functional of total potential energy. In the functional of [1] we did not take into account physical properties of the curvilinear interface itself such as, for example, the line tension of capillarity type and its possible generalizations. Also force and couple resultants acting along $C$ and at its intersections with $\partial M$ were not accounted for in [1]. To extend the functional of [1] by additional effects associated with $C$ let us remind some kinematical relations of the surface curve $C$ treated here as an axis of a virtual rod.

Within the dynamically and kinematically exact theory of regular rods developed in $[3,26]$ the initial (undeformed) placement $\kappa$ of the 3D rod-like body is represented by the position vector $\boldsymbol{x}_{C} \in E$ of the axis $C$ and three orthonormal vectors $\boldsymbol{\tau}_{\alpha}, \boldsymbol{\tau}$ attached to any point $x_{C} \in C$, with $\boldsymbol{\tau}=\mathrm{d} \boldsymbol{x}_{C} / \mathrm{d} s \equiv \boldsymbol{x}_{C}^{\prime}$. In the deformed placement $\gamma$ the rod is represented by the position vector $\boldsymbol{y}_{C} \in E$ of the material rod axis $D=\chi(C)$ and three attached orthonormal directors $\boldsymbol{h}_{\alpha}, \boldsymbol{h}$ which satisfy the relations

$$
\boldsymbol{y}_{C}=\chi\left(\boldsymbol{x}_{C}\right)=\boldsymbol{x}_{C}+\boldsymbol{u}_{C}, \quad \boldsymbol{h}_{\alpha}=\boldsymbol{R} \boldsymbol{\tau}_{\alpha}, \quad \boldsymbol{h}=\boldsymbol{R} \boldsymbol{\tau},
$$




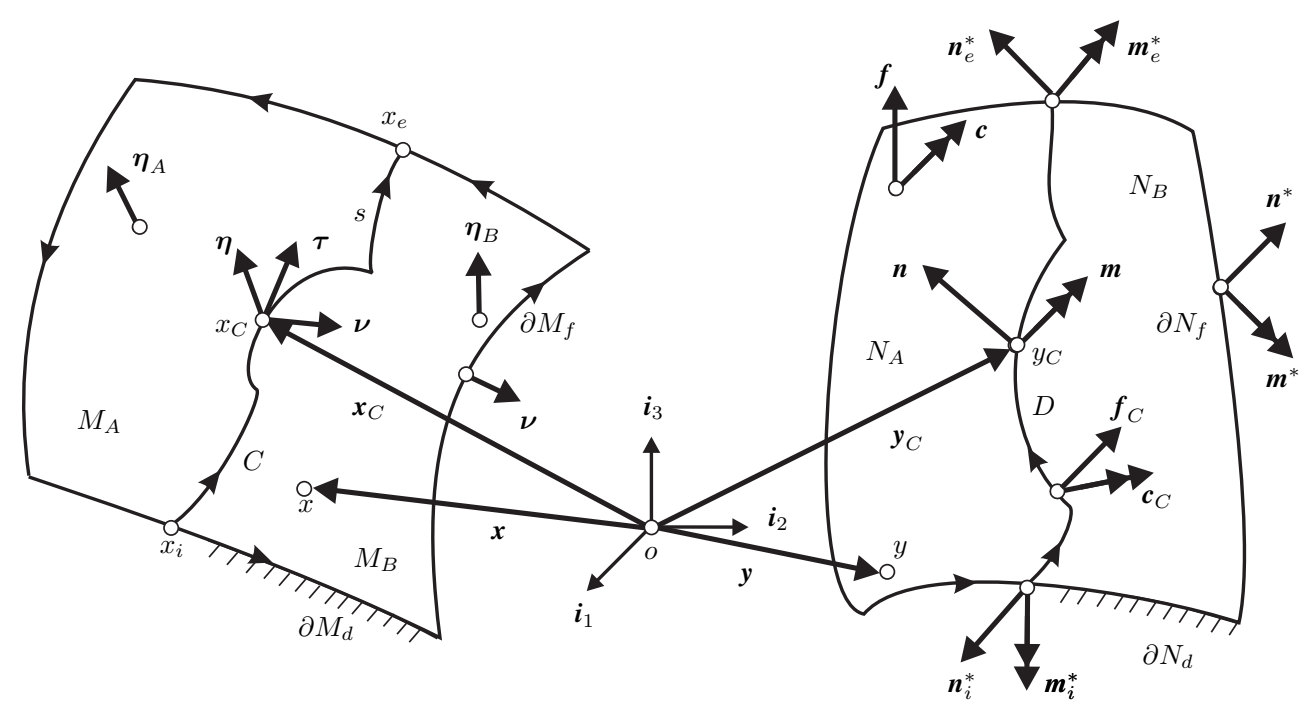

Fig. 1 Two-phase shell kinematics.

where $\boldsymbol{u}_{C}$ is the translation vector and $\boldsymbol{R}$ the rotation tensor describing the work-averaged gross deformation of the rod cross section.

The local deformation of the phase interface curve $C$ can be described by the natural strain $\varepsilon$ and bending $\kappa$ vectors in the spatial representation defined by $[3,26]$

$$
\boldsymbol{\varepsilon}=\boldsymbol{u}_{C}^{\prime}+(\mathbf{1}-\boldsymbol{R}) \boldsymbol{\tau}, \quad \kappa=a x\left(\boldsymbol{R}^{\prime} \boldsymbol{R}^{T}\right) .
$$

The 1D fields $\boldsymbol{u}_{C}(s), \boldsymbol{R}(s)$ along $C$ can be different, in general, from finite limits $\boldsymbol{u}^{ \pm}(s), \boldsymbol{Q}^{ \pm}(s)$ of the 2D fields $\boldsymbol{u}, \boldsymbol{Q}$ when the respective boundaries $\partial M_{A}$ or $\partial M_{B}$ coinciding with $C$ are approached. As a result, each type of the phase transition should be characterised by additional relations between $\boldsymbol{u}_{C}, \boldsymbol{R}$ and $\boldsymbol{u}^{ \pm}, \boldsymbol{Q}^{ \pm}$. In this paper we discuss only such types of PT which do not lead to fragmentation of the shell. This is possible in two types of PT.

The phase interface $C$ is called coherent if both fields $\boldsymbol{y}$ and $\boldsymbol{Q}$ are continuous at $C$

$$
\begin{gathered}
\boldsymbol{y}^{-}=\boldsymbol{y}^{+}=\boldsymbol{y}_{C}, \quad \llbracket \boldsymbol{y} \rrbracket=0, \quad \llbracket \boldsymbol{y}^{\prime} \rrbracket=\mathbf{0}, \\
\boldsymbol{Q}^{-}=\boldsymbol{Q}^{+}=\boldsymbol{R}, \quad \llbracket \boldsymbol{Q} \rrbracket=\mathbf{0}, \quad \llbracket \boldsymbol{Q}^{\prime} \rrbracket=\mathbf{0},
\end{gathered}
$$

where $\llbracket \ldots \rrbracket=(\ldots)^{+}-(\ldots)^{-}$is the jump at $C$. Therefore, the coherent curve $C$ can be singular with regard to $\boldsymbol{F}=\operatorname{Grad}_{s} \boldsymbol{y}$ and $\operatorname{Grad}_{s} \boldsymbol{Q}$ but not with regard to $\boldsymbol{y}$ and $\boldsymbol{Q}$ themselves, where $\operatorname{Grad}_{s}$ is the surface gradient operator defined intrinsically in $[27,28]$.

The phase interface is called incoherent in rotations if $\boldsymbol{y}$ is continuous at $C$ but continuity of $\boldsymbol{Q}$ at $C$ is violated. In this case the conditions indicated in the first row of (6) are still satisfied, but those indicated in the second row of (6) can be violated. Therefore, the interface $C$ incoherent in rotations can be singular with regard to $\boldsymbol{F}, \operatorname{Grad}_{s} \boldsymbol{Q}$, and $\boldsymbol{Q}$.

\section{Weak formulation of equilibrium BVP}

The equilibrium BVP for shells with occurrence of the PT can now be formulated in the following weak form extending the one proposed in $[1,29]$ :

Given the external, resultant surface force and couple fields $\boldsymbol{f}\left(\theta^{\alpha}\right), \boldsymbol{c}\left(\theta^{\alpha}\right)$ on $M$, curvilinear force and couple fields $\boldsymbol{f}_{C}(s)$, $\boldsymbol{c}_{C}(s)$ along $C$, concentrated forces and couples $\boldsymbol{n}_{i}^{*}, \boldsymbol{m}_{i}^{*}$ and $\boldsymbol{n}_{e}^{*}, \boldsymbol{m}_{e}^{*}$ acting at initial and end points $x_{i}, x_{e}$ of intersection of $C$ with $\partial M_{f}$, as well as boundary force and couple fields $\boldsymbol{n}^{*}(s), \boldsymbol{m}^{*}(s)$ prescribed along $\partial M_{f}$, respectively, find a solution $\left(\boldsymbol{u}, \boldsymbol{Q}, \boldsymbol{x}_{C}\right)$ on the configuration space $S(M ; E \times S O(3) \times E)$ satisfying the kinematic boundary conditions $\boldsymbol{u}=\boldsymbol{u}^{*}, \boldsymbol{Q}=\boldsymbol{Q}^{*}$ along $\partial M_{d}=\partial M \backslash \partial M_{f}$ such that for any kinematically admissible virtual vector fields $\delta \boldsymbol{u}, \boldsymbol{w}=a x\left(\delta \boldsymbol{Q} \boldsymbol{Q}^{T}\right), \delta \boldsymbol{x}_{C}$ the following variational principle of the total potential energy is satisfied:

$$
\delta I=0, \quad I=\iint_{M_{A}} W_{A} \mathrm{~d} a+\iint_{M_{B}} W_{B} \mathrm{~d} a+\int_{C} W_{C} \mathrm{~d} s-A .
$$


Here $W_{A}=W_{A}(\boldsymbol{E}, \boldsymbol{K})$ and $W_{B}=W_{B}(\boldsymbol{E}, \boldsymbol{K})$ are the 2D elastic strain energy densities associated with the subregions $M_{A}$ and $M_{B}$, respectively, $W_{C}=W_{C}(\varepsilon, \kappa)$ is the 1 D elastic strain energy density associated with the phase interface curve $C$, and $A$ is the potential of external loads such that

$$
\begin{aligned}
\delta A= & \iint_{M \backslash C}(\boldsymbol{f} \cdot \delta \boldsymbol{u}+\boldsymbol{c} \cdot \boldsymbol{w}) \mathrm{d} a+\int_{C}\left(\boldsymbol{f}_{C} \cdot \delta \boldsymbol{u}_{C}+\boldsymbol{c}_{C} \cdot \boldsymbol{w}_{C}\right) \mathrm{d} s \\
& +\int_{\partial M_{f}}\left(\boldsymbol{n}^{*} \cdot \delta \boldsymbol{u}+\boldsymbol{m}^{*} \cdot \boldsymbol{w}\right) \mathrm{d} s+\boldsymbol{n}_{e}^{*} \cdot \delta \boldsymbol{u}_{e}+\boldsymbol{m}_{e}^{*} \cdot \boldsymbol{w}_{e}-\boldsymbol{n}_{i}^{*} \cdot \delta \boldsymbol{u}_{i}-\boldsymbol{m}_{i}^{*} \cdot \boldsymbol{w}_{i} .
\end{aligned}
$$

In (8), $\delta \boldsymbol{u}_{C}$ and $\boldsymbol{w}_{C}=a x\left(\delta \boldsymbol{R} \boldsymbol{R}^{T}\right)$ are the virtual translation and rotation of the interface $C$, and corresponding virtual displacements at the initial and end points of $C$ are denoted by $\delta \boldsymbol{u}_{i}, \boldsymbol{w}_{i}$ and $\delta \boldsymbol{u}_{e}, \boldsymbol{w}_{e}$, respectively.

The principle (7) $)_{1}$ with (7) 2 and (8) states that among all possible values of $\boldsymbol{u}, \boldsymbol{Q}$ in $M \backslash C, \boldsymbol{u}_{C}, \boldsymbol{R}$ along $C$, and positions of the phase interface $\boldsymbol{x}_{C}$ the actual solution renders the functional (7) $)_{2}$ stationary.

Calculating $\delta I$ with $I$ defined by $(7)_{2}$ consists of two parts. In the first part we have to repeat all transformations given in detail in [1] for $I$ without the integral over $C$ in $(7)_{2}$ as well as without the integral over $C$ and out of integral terms in (8). Due to the limited volume of this paper we do not repeat here those transformations and refer the reader to the final result given in [1], f. (26).

Variation of the additional integral in (7) $)_{2}$ performed with the help of (5) and the theorem for differentiation of curvilinear integrals [13] (f. (1.158), (2.223)) or [30] (f. 24) with a movable surface curve leads to

$$
\begin{aligned}
\delta \int_{C} W_{C} \mathrm{~d} s & =\int_{C} \delta W_{C} \mathrm{~d} s+\int_{C} k_{g} V W_{C} \mathrm{~d} s \\
& =\int_{C}\left(\boldsymbol{n} \cdot \delta^{c} \boldsymbol{\varepsilon}+\boldsymbol{m} \cdot \delta^{c} \boldsymbol{\kappa}\right) \mathrm{d} s+\int_{C} k_{g} V W_{C} \mathrm{~d} s \\
& =\int_{C}\left\{\boldsymbol{n} \cdot\left(\delta \boldsymbol{u}_{C}^{\prime}-\boldsymbol{w}_{C} \times \boldsymbol{y}_{C}^{\prime}\right)+\boldsymbol{m} \cdot \boldsymbol{w}_{C}^{\prime}\right\} \mathrm{d} s+\int_{C} k_{g} V W_{C} \mathrm{~d} s \\
& =\left.\left(\boldsymbol{n} \cdot \delta \boldsymbol{u}_{C}+\boldsymbol{m} \cdot \boldsymbol{w}_{C}\right)\right|_{x_{i}} ^{x_{e}}-\int_{C}\left\{\boldsymbol{n}^{\prime} \cdot \delta \boldsymbol{u}_{C}+\left(\boldsymbol{m}^{\prime}+\boldsymbol{y}_{C}^{\prime} \times \boldsymbol{n}\right) \cdot \boldsymbol{w}_{C}\right\} \mathrm{d} s+\int_{C} k_{g} V W_{C} \mathrm{~d} s,
\end{aligned}
$$

where $\delta^{c}$ is the corotational variation, $k_{g}=-\frac{\partial \boldsymbol{\tau}}{\partial s} \cdot \boldsymbol{\nu}$ is the geodesic curvature of $C, \boldsymbol{\nu}$ is the external unit normal to $\partial M$, $V=\delta \boldsymbol{x}_{C} \cdot \boldsymbol{\nu}$, and we have used the relations for $\delta^{c} \boldsymbol{\varepsilon}$ and $\delta^{c} \boldsymbol{\kappa}$ given in [3]. Because we are interested in the continuity conditions along $C$ we assume here that $\delta \boldsymbol{x}_{C}=\mathbf{0}$ at the end points of $C$ to avoid discussion of the continuity conditions at the singular points which we feel to be of secondary importance in this problem. We may also consider the closed curve $C$. The stress resultant $\boldsymbol{n}$ and stress couple $\boldsymbol{m}$ vectors in the spatial representation are given by the constitutive equations

$$
\boldsymbol{n}=\frac{\partial W_{C}}{\partial \varepsilon}, \boldsymbol{m}=\frac{\partial W_{C}}{\partial \boldsymbol{\kappa}} .
$$

Introducing (9) and (8) into (7) ${ }_{1}$ and using the results of transformations given in [1], f. (26) we obtain

$$
\begin{aligned}
\delta I= & -\iint_{M \backslash C}\left\{\left(\left.\boldsymbol{n}^{\alpha}\right|_{\alpha}+\boldsymbol{f}\right) \cdot \delta \boldsymbol{u}+\left(\left.\boldsymbol{m}^{\alpha}\right|_{\alpha}+\boldsymbol{y},_{\alpha} \times \boldsymbol{n}^{\alpha}+\boldsymbol{c}\right) \cdot \boldsymbol{w}\right\} \mathrm{d} a \\
& +\int_{\partial M_{f}}\left\{\left(\boldsymbol{n}_{\nu}-\boldsymbol{n}^{*}\right) \cdot \delta \boldsymbol{u}+\left(\boldsymbol{m}_{\nu}-\boldsymbol{m}^{*}\right) \cdot \boldsymbol{w}\right\} \mathrm{d} s \\
& +\int_{\partial M_{d}}\left(\boldsymbol{n}_{\nu} \cdot \delta \boldsymbol{u}+\boldsymbol{m}_{\nu} \cdot \boldsymbol{w}\right) \mathrm{d} s \\
& -\int_{C}\left\{V \llbracket W \rrbracket-k_{g} V W_{C}+\llbracket \boldsymbol{n}_{\nu} \cdot \delta \boldsymbol{u} \rrbracket+\llbracket \boldsymbol{m}_{\nu} \cdot \boldsymbol{w} \rrbracket\right. \\
& \left.\quad+\left(\boldsymbol{n}^{\prime}+\boldsymbol{f}_{C}\right) \cdot \delta \boldsymbol{u}_{C}+\left(\boldsymbol{m}^{\prime}+\boldsymbol{y}_{C}^{\prime} \times \boldsymbol{n}+\boldsymbol{c}_{C}\right) \cdot \boldsymbol{w}_{C}\right\} \mathrm{d} s \\
& +\left(\boldsymbol{n}_{e}-\boldsymbol{n}_{e}^{*}\right) \cdot \delta \boldsymbol{u}_{e}+\left(\boldsymbol{m}_{e}-\boldsymbol{m}_{e}^{*}\right) \cdot \boldsymbol{w}_{e}-\left(\boldsymbol{n}_{i}-\boldsymbol{n}_{i}^{*}\right) \cdot \delta \boldsymbol{u}_{i}-\left(\boldsymbol{m}_{i}-\boldsymbol{m}_{i}^{*}\right) \cdot \boldsymbol{w}_{i}=0,
\end{aligned}
$$


where

$$
\boldsymbol{n}^{\alpha}=\frac{\partial W}{\partial \boldsymbol{\varepsilon}_{\alpha}}, \quad \boldsymbol{m}^{\alpha}=\frac{\partial W}{\partial \boldsymbol{\kappa}_{\alpha}}, \quad \boldsymbol{n}_{\nu}=\boldsymbol{N} \boldsymbol{\nu}=\boldsymbol{n}^{\alpha} \nu_{\alpha}, \quad \boldsymbol{m}_{\nu}=\boldsymbol{M} \boldsymbol{\nu}=\boldsymbol{m}^{\alpha} \nu_{\alpha},
$$

$\nu_{\alpha}=\boldsymbol{\nu} \cdot \boldsymbol{a}_{\alpha}$, and $\boldsymbol{N}, \boldsymbol{M}$ are the stress resultant and stress couple tensors of the Kirchhoff type, respectively.

Vanishing of the first two rows of (11) leads to known local equilibrium equations and natural boundary conditions of the general theory of regular shells, see [1]. The third and sixth rows of (11) vanish identically if the kinematic boundary conditions along $\partial M_{d}$ are satisfied, for then $\delta \boldsymbol{u}=\boldsymbol{w}=\delta \boldsymbol{u}_{e}=\boldsymbol{w}_{e}=\delta \boldsymbol{u}_{i}=\boldsymbol{w}_{i}=\mathbf{0}$ along $\partial M_{d}$.

\section{Extended natural continuity conditions}

From (11) it also follows that at the curvilinear phase interface the following local, extended, static natural continuity conditions have to be satisfied:

$$
\begin{aligned}
& V \llbracket W \rrbracket-k_{g} V W_{C}+\llbracket \boldsymbol{n}_{\nu} \cdot \delta \boldsymbol{u} \rrbracket+\llbracket \boldsymbol{m}_{\nu} \cdot \boldsymbol{w} \rrbracket+\left(\boldsymbol{n}^{\prime}+\boldsymbol{f}_{C}\right) \cdot \delta \boldsymbol{u}_{C}+\left(\boldsymbol{m}^{\prime}+\boldsymbol{y}_{C}^{\prime} \times \boldsymbol{n}+\boldsymbol{c}_{C}\right) \cdot \boldsymbol{w}_{C}=0 \quad \text { along } C, \\
& \boldsymbol{n}_{i}=\boldsymbol{n}_{i}^{*}, \quad \boldsymbol{m}_{i}=\boldsymbol{m}_{i}^{*} \text { at } x_{i}=C \cap \partial M_{f}, \\
& \boldsymbol{n}_{e}=\boldsymbol{n}_{e}^{*}, \quad \boldsymbol{m}_{e}=\boldsymbol{m}_{e}^{*} \text { at } x_{e}=C \cap \partial M_{f} .
\end{aligned}
$$

The fields $V, \delta \boldsymbol{u}^{ \pm}, \boldsymbol{w}^{ \pm}, \delta \boldsymbol{u}_{C}, \boldsymbol{w}_{C}$ in (13) are still not independent at $C$, in general. Performing more detailed analysis of (13) for different types of PTs we can introduce additional information about the behavior of the virtual fields and their interrelation.

Assuming that $\boldsymbol{y}(x)$ and $\boldsymbol{Q}(x)$ are smooth fields in the interiors of $M_{A}$ and $M_{B}$ we can define one-sided limits $\boldsymbol{y}^{ \pm}, \boldsymbol{F}^{ \pm}$, $\boldsymbol{Q}^{ \pm},\left(\operatorname{Grad}_{s} \boldsymbol{Q}\right)^{ \pm}$when $\boldsymbol{x} \rightarrow \boldsymbol{x}_{C}$. The functions $\boldsymbol{y}^{ \pm}(s)$ and $\boldsymbol{Q}^{ \pm}(s)$ are continuously differentiable along $C$.

\subsection{Coherent interface}

For the coherent interface all the relations (6) are assumed to be satisfied. Then applying the Maxwell theorem [31] we obtain the local kinematic compatibility conditions [1]

$$
\begin{aligned}
& \llbracket \delta \boldsymbol{u} \rrbracket+V \llbracket \boldsymbol{F} \boldsymbol{\nu} \rrbracket=\mathbf{0}, \quad \llbracket \boldsymbol{w} \rrbracket+V \llbracket \boldsymbol{K} \boldsymbol{\nu} \rrbracket=\mathbf{0} \quad \text { along } C, \\
& \boldsymbol{F} \boldsymbol{\nu}=\boldsymbol{y},_{\alpha} \nu^{\alpha}, \quad \boldsymbol{K} \boldsymbol{\nu}=\boldsymbol{\kappa}_{\alpha} \nu^{\alpha},
\end{aligned}
$$

which allow one to relate $\llbracket \delta \boldsymbol{u} \rrbracket$ and $\llbracket \boldsymbol{w} \rrbracket$ with $V$.

Let us represent the jumps of products in (13) by the identities

$$
\llbracket N \nu \cdot \delta u \rrbracket=\langle\boldsymbol{N} \nu\rangle \cdot \llbracket \delta u \rrbracket+\llbracket N \nu \rrbracket \cdot\langle\delta u\rangle, \quad \llbracket M \nu \cdot w \rrbracket=\langle M \nu\rangle \cdot \llbracket w \rrbracket+\llbracket M \nu \rrbracket \cdot\langle w\rangle .
$$

Introducing (15) and (17) into (13) we obtain

$$
\begin{aligned}
& V\left\{\llbracket W \rrbracket-k_{g} W_{C}-\langle\boldsymbol{N} \boldsymbol{\nu}\rangle \cdot \llbracket \boldsymbol{F} \boldsymbol{\nu} \rrbracket-\langle\boldsymbol{M} \boldsymbol{\nu}\rangle \cdot \llbracket \boldsymbol{K} \boldsymbol{\nu} \rrbracket\right\}+\llbracket \boldsymbol{N} \boldsymbol{\nu} \rrbracket \cdot\langle\delta \boldsymbol{u}\rangle+\llbracket \boldsymbol{M} \boldsymbol{\nu} \rrbracket \cdot\langle\boldsymbol{w}\rangle \\
& +\left(\boldsymbol{n}^{\prime}+\boldsymbol{f}_{C}\right) \cdot \delta \boldsymbol{u}_{C}+\left(\boldsymbol{m}^{\prime}+\boldsymbol{y}_{C}^{\prime} \times \boldsymbol{n}+\boldsymbol{c}_{C}\right) \cdot \boldsymbol{w}_{C}=0 .
\end{aligned}
$$

At the coherent interface $C$ from (6) it follows that $\langle\delta \boldsymbol{u}\rangle=\delta \boldsymbol{u}_{C}$ and $\langle\boldsymbol{w}\rangle=\boldsymbol{w}_{C}$. Therefore

$$
\begin{aligned}
& V\left\{\llbracket W \rrbracket-k_{g} W_{C}-\langle\boldsymbol{N} \boldsymbol{\nu}\rangle \cdot \llbracket \boldsymbol{F} \boldsymbol{\nu} \rrbracket-\langle\boldsymbol{M} \boldsymbol{\nu}\rangle \cdot \llbracket \boldsymbol{K} \boldsymbol{\nu} \rrbracket\right\} \\
& +\left(\boldsymbol{n}^{\prime}+\llbracket \boldsymbol{N} \boldsymbol{\nu} \rrbracket+\boldsymbol{f}_{C}\right) \cdot \delta \boldsymbol{u}_{C}+\left(\boldsymbol{m}^{\prime}+\boldsymbol{y}_{C}^{\prime} \times \boldsymbol{n}+\llbracket \boldsymbol{M} \boldsymbol{\nu} \rrbracket+\boldsymbol{c}_{C}\right) \cdot \boldsymbol{w}_{C}=0 .
\end{aligned}
$$

For now independent virtual fields $V, \delta \boldsymbol{u}_{C}$, and $\boldsymbol{w}_{C}$ the relation (19) requires that the following local continuity conditions be satisfied along $C$ :

$$
\begin{aligned}
& \llbracket W \rrbracket-\langle\boldsymbol{N} \boldsymbol{\nu}\rangle \cdot \llbracket \boldsymbol{F} \boldsymbol{\nu} \rrbracket-\langle\boldsymbol{M} \boldsymbol{\nu}\rangle \cdot \llbracket \boldsymbol{K} \boldsymbol{\nu} \rrbracket=k_{g} W_{C}, \\
& \boldsymbol{n}^{\prime}+\llbracket \boldsymbol{N} \boldsymbol{\nu} \rrbracket+\boldsymbol{f}_{C}=\mathbf{0}, \quad \boldsymbol{m}^{\prime}+\boldsymbol{y}_{C}^{\prime} \times \boldsymbol{n}+\llbracket \boldsymbol{M} \boldsymbol{\nu} \rrbracket+\boldsymbol{c}_{C}=\mathbf{0} .
\end{aligned}
$$

The continuity conditions (14) and (20) are more complex than those derived in [1], f. (37) $)_{1,2}$ and (39), because now they bring additional information about the elastic strain energy associated with the phase interface itself as well as the resultant forces and couples acting along $C$ and at the initial $x_{i}$ and end $x_{e}$ points of $C$. The conditions (14) and (20) $)_{2,3}$ are formally the same as the ones of the exact, resultant, static continuity conditions of the branching and intersecting shells [25] derived by direct through-the-thickness integration of equilibrium conditions of continuum mechanics. This suggests that the conditions (14) and (20) can also be formulated exactly by direct through-the-thickness integration of appropriate 3D equilibrium conditions of the shell-like body undergoing PT in a thin tube of matter about the singular surface curve $C$. 


\subsection{Interface incoherent in rotations}

For the interface incoherent in rotations only the relations of the first row of (6) are assumed to be satisfied. In this case the interface $C$ can be singular with regard to $\boldsymbol{Q}, \boldsymbol{F}$, and $\operatorname{Grad}_{s} \boldsymbol{Q}$. From the Maxwell theorem [31] follows only the first compatibility condition (6) ${ }_{1}$, while the second one (6) $)_{2}$ can now be violated, in general. Here $\langle\delta \mathbf{u}\rangle=\delta \boldsymbol{u}_{C}$ as well, but $\langle\boldsymbol{w}\rangle \neq \boldsymbol{w}_{C}$, in general. Then the continuity condition (13) can be transformed into

$$
\begin{aligned}
& V\left\{\llbracket W \rrbracket-k_{g} W_{C}-\langle\boldsymbol{N} \boldsymbol{\nu}\rangle \cdot \llbracket \boldsymbol{F} \boldsymbol{\nu} \rrbracket\right\}+\llbracket \boldsymbol{M} \boldsymbol{\nu} \cdot \boldsymbol{w} \rrbracket \\
& +\left(\boldsymbol{n}^{\prime}+\llbracket \boldsymbol{N} \boldsymbol{\nu} \rrbracket+\boldsymbol{f}_{C}\right) \cdot \delta \boldsymbol{u}_{C}+\left(\boldsymbol{m}^{\prime}+\boldsymbol{y}_{C}^{\prime} \times \boldsymbol{n}+\boldsymbol{c}_{C}\right) \cdot \boldsymbol{w}_{C}=0 .
\end{aligned}
$$

With now independent virtual fields $V, \boldsymbol{w}^{ \pm}, \delta \boldsymbol{u}_{C}, \boldsymbol{w}_{C}$ the relation (21) is equivalent to the following set of local, static, natural continuity conditions along the interface $C$ incoherent in rotations:

$$
\begin{aligned}
& \llbracket W \rrbracket-\langle\boldsymbol{N} \boldsymbol{\nu}\rangle \cdot \llbracket \boldsymbol{F} \boldsymbol{\nu} \rrbracket=k_{g} W_{C}, \quad \boldsymbol{M}^{ \pm} \boldsymbol{\nu}=\mathbf{0}, \\
& \boldsymbol{n}^{\prime}+\llbracket \boldsymbol{N} \rrbracket+\boldsymbol{f}_{C}=\mathbf{0}, \quad \boldsymbol{m}^{\prime}+\boldsymbol{y}_{C}^{\prime} \times \boldsymbol{n}+\boldsymbol{c}_{C}=\mathbf{0} .
\end{aligned}
$$

The second conditions in the first row of (22) indicate that here there appears the kind of a hinge along $C$ at which the resultant surface couples should vanish. The conditions (22) express just the balance of forces and couples at the interface $C$ incoherent in rotations.

The static natural continuity conditions $(20)_{1}$ and $(22)_{1}$ are the relations which allow one to establish position of the singular curve $C$ on $M$ in the thermodynamic equilibrium state of phase transition.

\subsection{Capillary energy of the phase interface}

Let us consider a special case of curvilinear energy of the phase interface by analogy to the one used in the theory of capillary surfaces [32,33]. In our 1D case the therm responsible for curvilinear energy in the functional (7) can be assumed to be given by the integral along $D$

$$
\sigma \int_{D} \mathrm{~d} \bar{s}, \text { where } \mathrm{d} \bar{s}=\sqrt{\boldsymbol{y}_{C}^{\prime} \cdot \boldsymbol{y}_{C}^{\prime}} \mathrm{d} s, \text { therefore } W_{C}=\sigma \sqrt{\boldsymbol{y}_{C}^{\prime} \cdot \boldsymbol{y}_{C}^{\prime}} .
$$

Here $\sigma$ is the constant line tension, while $\bar{s}$ is the length parameter along $D$. Other possible types of constitutive equations of one-dimensional continua are discussed in [13]. The concept of line tension is widely used not only in the theory of capillarity but also in the theory of dislocations where the line tension describes the energy of a tube surrounding the dislocation, see [34]. For the curvilinear energy (23) we can simplify the continuity conditions along the phase interface (20) and (22). Indeed, if we use (10) then from the equation (23) we obtain

$$
\boldsymbol{n}=\frac{\sigma}{\sqrt{\boldsymbol{y}_{C}^{\prime} \cdot \boldsymbol{y}_{C}^{\prime}}} \boldsymbol{y}_{C}^{\prime}, \quad \boldsymbol{m}=\mathbf{0}
$$

Thus, using (24) we obtain that $\boldsymbol{n} \times \boldsymbol{y}_{C}^{\prime}=\mathbf{0}$. If we further assume that $\boldsymbol{c}_{C}=\mathbf{0}$ the third equation of (20) reduces to $\llbracket \boldsymbol{M} \boldsymbol{\nu} \rrbracket=\mathbf{0}$ while the fourth one of (22) is satisfied identically.

Note that $\frac{1}{\sqrt{y_{C}^{\prime} \cdot y_{C}^{\prime}}} y_{C}^{\prime}$ is the unit vector tangent to the curve $D$. Then using the Frenet formulas we obtain that $\left(\frac{1}{\sqrt{\boldsymbol{y}_{C}^{\prime} \cdot \boldsymbol{y}_{C}^{\prime}}} \boldsymbol{y}_{C}^{\prime}\right)^{\prime}=k \boldsymbol{\mu}$, where $k$ is the principal curvature of the curve $D$ and $\boldsymbol{\mu}$ is the principal unit normal to $D$. Then with the additional assumption that $\boldsymbol{f}_{C}=\mathbf{0}$ the second equation of (20) or the third one of (22) reduces to

$$
\sigma k \boldsymbol{\mu}+\llbracket \boldsymbol{N} \boldsymbol{\nu} \rrbracket=\mathbf{0} .
$$

The equation (25) is a 1D analog of the well-known Laplace equation in the theory of capillarity $[32,33]$.

\section{Example}

Let us solve a simple axisymmetric plane problem of PT in an infinite plate with a central hole of radius $a$ taking into account the curvilinear energy given by (23), Fig. 2. This example was considered in [1] in the case $\sigma=0$. It is assumed that in 

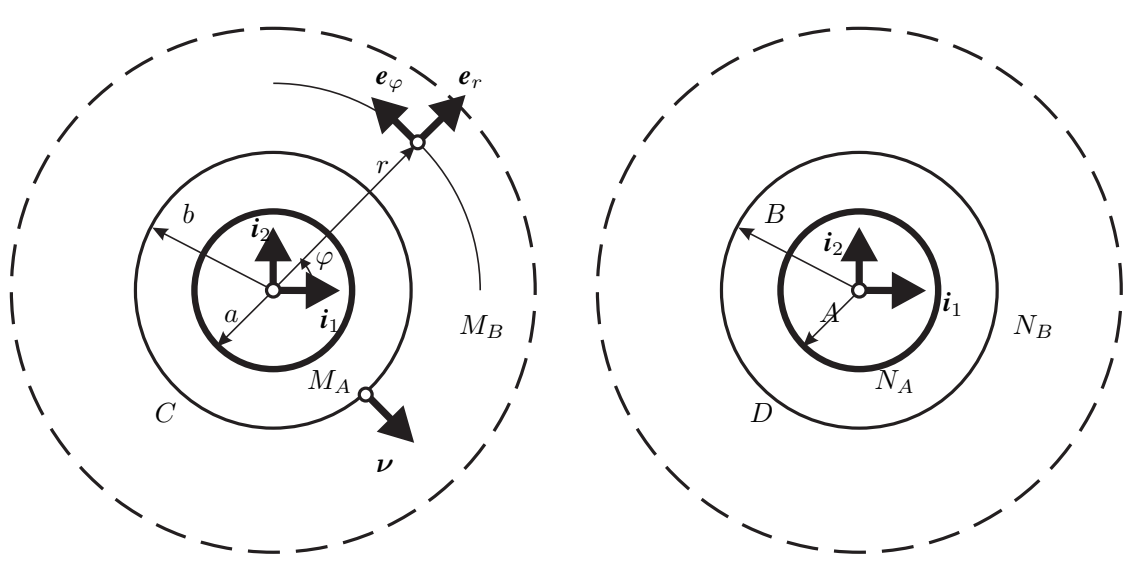

Fig. 2 Two-phase plate with a hole.

the undeformed state the whole plate consists of one material phase marked by " $B$ ". Then to the hole boundary the radial translation $\boldsymbol{u}^{*}=-u^{*} \boldsymbol{e}_{r}$ with a constant magnitude $u^{*}$ is prescribed. As a result, in the deformed equilibrium state of phase transition there may appear in a region $a<r<b$ a new material phase marked by " $A$ ". Our goal is to find the radius $b$ of the interface circle $C$.

In the polar coordinate system $\theta^{1}=r, \theta^{2}=\varphi$ the axisymmetric plane deformation and corresponding strain and bending measures are given by

$$
\begin{aligned}
& \boldsymbol{y}=y(r) \boldsymbol{e}_{r}, \quad \boldsymbol{u}=u(r) \boldsymbol{e}_{r}=(y(r)-r) \boldsymbol{e}_{r}, \quad \boldsymbol{Q}=\mathbf{1}, \quad \boldsymbol{y}_{C}=B \boldsymbol{e}_{r}, \quad \boldsymbol{x}_{C}=b \boldsymbol{e}_{r}, \\
& \boldsymbol{e}_{r}=\cos \varphi \boldsymbol{i}_{1}+\sin \varphi \boldsymbol{i}_{2}, \quad \boldsymbol{e}_{\varphi}=-\sin \varphi \boldsymbol{i}_{1}+\cos \varphi \boldsymbol{i}_{2}, \\
& \boldsymbol{\varepsilon}_{r}=\frac{d u}{d r} \boldsymbol{e}_{r} \equiv u^{\prime} \boldsymbol{e}_{r}, \quad \varepsilon_{\varphi}=u \boldsymbol{e}_{\varphi}, \quad \boldsymbol{\kappa}_{r}=\boldsymbol{\kappa}_{\varphi} \equiv \mathbf{0}, \\
& \boldsymbol{E}=E_{r} \boldsymbol{e}_{r} \otimes \boldsymbol{e}_{r}+E_{\varphi} \boldsymbol{e}_{\varphi} \otimes \boldsymbol{e}_{\varphi}, \quad E_{r}=u^{\prime}, \quad E_{\varphi}=\frac{u}{r}
\end{aligned}
$$

where $\boldsymbol{e}_{r}$ and $\boldsymbol{e}_{\varphi}$ are the unit base vectors, $E_{r}$ and $E_{\varphi}$ are the physical components of strain, while $B$ or $b$ and $y(r)$ or $u(r)$ are unknowns of the BVP.

To avoid awkward computations of [1] the elastic strain energy densities $W_{A}$ and $W_{B}$ are assumed in the simple form

$$
W_{A, B}=C_{1}^{A, B}\left(E_{r}^{2}+E_{\varphi}^{2}\right)+\Delta_{A, B} \equiv C_{1}^{A, B}\left(u^{\prime 2}+\frac{u^{2}}{r^{2}}\right)+\Delta_{A, B},
$$

where $\Delta_{A, B}$ is a constant. Usually we determine the strain energy density with accuracy to a constant, but here we may take into account that the reference values of the densities of the two phases at zero strains may be different, in general. If we assume that $\Delta_{B}=0$, then $\Delta_{A}$ describes an energetic barrier for the formation of the new phase " $A$ ". Further we take $\Delta_{A} \equiv C_{1}^{A} \Delta \neq 0$ where $\Delta$ is a dimensionless parameter. The one-constant constitutive equation (27) means that the strain energy densities of two material phases differ only by values of elastic constants $C_{1}^{A, B}$ while other elastic constants in the constitutive equation of isotropic shell discussed in [1] are equal zero.

For the deformation (26) and the constitutive equations (24) and (27) we have

$$
\begin{aligned}
& \boldsymbol{N}_{A, B}=N_{r}^{A, B} \boldsymbol{e}_{r} \otimes \boldsymbol{e}_{r}+N_{\varphi}^{A, B} \boldsymbol{e}_{\varphi} \otimes \boldsymbol{e}_{\varphi}, \quad \boldsymbol{M}_{A, B} \equiv \mathbf{0}, \quad \boldsymbol{n}=\sigma \boldsymbol{e}_{\varphi}, \quad \boldsymbol{m}=\mathbf{0}, \quad W_{C}=\sigma \frac{B}{b}, \\
& N_{r}^{A, B}=2 C_{1}^{A, B} u^{\prime}, \quad N_{\varphi}^{A, B}=2 C_{1}^{A, B} \frac{u}{r} .
\end{aligned}
$$

Solving the equilibrium equations we obtain [1]

$$
u_{A, B}(r)=u_{0}^{A, B} r+\frac{u_{1}^{A, B}}{r},
$$

where $u_{0}^{A, B}$ and $u_{1}^{A, B}$ are integration constants.

Using kinematic boundary conditions and kinematic continuity conditions

$$
u_{A}(a)=u^{*}, \quad u_{A}(b)=u_{B}(b), \quad \text { and } u_{B}(r) \rightarrow 0 \text { for } r \rightarrow \infty,
$$


we obtain that $u_{0}^{B}=0, u_{1}^{B}=u_{1}^{A}+b^{2} u_{0}^{A}$, and $u_{0}^{A}=\frac{u^{*}}{a}-\frac{u_{1}^{A}}{a^{2}}$. The integration constant $u_{1}^{A}$ can be found from the reduced third equation (22):

$$
\llbracket \boldsymbol{N} \boldsymbol{\nu} \equiv\left[N_{r}^{A}(b)-N_{r}^{B}(b)\right] \boldsymbol{e}_{r}=\frac{\sigma}{b} \boldsymbol{e}_{r},
$$

while the a priory unknown radius $b$ of the phase interface circle $C$ from the first of equations (20) or (22):

$$
C_{1}^{A}\left[u_{A}^{\prime 2}(b)+\frac{u_{A}^{2}(b)}{b^{2}}\right]-C_{1}^{B}\left[u_{B}^{\prime 2}(b)+\frac{u_{B}^{2}(b)}{b^{2}}\right]+\Delta_{A}-\frac{1}{2}\left[N_{r}^{A}(b)+N_{r}^{B}(b)\right]\left[u_{A}^{\prime}(b)-u_{B}^{\prime}(b)\right]=\frac{\sigma B}{b^{2}} .
$$

The two-phase solution exists only if $u^{*}>u_{0}^{*}$, where $u_{0}^{*}=a \frac{\bar{\sigma} c+2 \sqrt{\bar{\sigma} c^{2}-\bar{\sigma}-\Delta+\bar{\sigma}^{2}+\Delta c^{2}}}{2\left(c^{2}-1\right)}, c=\frac{C_{1}^{B}}{C_{1}^{A}}>0$, and $\bar{\sigma}=\frac{\sigma}{C_{1}^{A} a}$. The solution indicates that within $0<u^{*}<u_{0}^{*}$ there is only one material phase " $B$ " in the plate. When the assumed boundary translation reaches the critical value $u^{*}=u_{0}^{*}$ the second more "soft" material phase " $A$ " is created with a smaller modulus of elasticity than that of the phase " $B$ ". Finally, when $u^{*}>u_{0}^{*}$ the plate consists of two regions with two different material phases separated by the circular interface $C$ of radius $b$. The value of $b$ depends upon the ratio $c$ between the material constants $C_{1}^{B}$ and $C_{1}^{A}$, as well as upon $\bar{\sigma}$ and $\Delta$ (see Fig. 3).

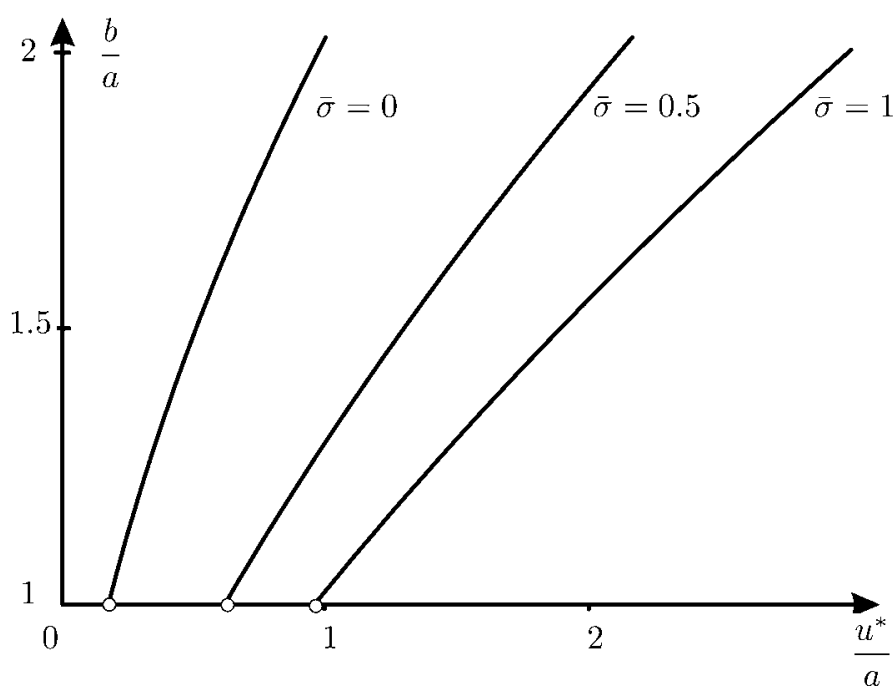

Fig. 3 Phase diagram for two-phase plate.

Note that in this problem the line tension affects position of the phase interface $C$ if the value of $\bar{\sigma}$ is big enough. If $\bar{\sigma} \ll 1$ then in the limit $\bar{\sigma} \rightarrow 0$ we obtain the results given in [1]. In the theory of phase transitions of 3D elastic bodies [10] the surface tension essentially affects the new phase nucleation if the curvature of nucleation surface is big enough. In the example considered here the nucleation curve of the new phase is a ring which curvature is not so big to affect the phase transition for small values of the line tension.

After determining the integration constants the total energy $I$ is a function of parameters $x \equiv b / a$ and $\bar{u} \equiv u^{*} / a$ and is given by the relation

$$
\begin{aligned}
\frac{1}{2 \pi a^{2} C_{1}^{A}} I(x, \bar{u})= & \frac{1}{c x^{2}+x^{2}-c+1}\left\{2 \bar{u}^{2}\left(x^{2}+c x^{2}+c-1\right)+2 \bar{u} \bar{\sigma} x\right. \\
& +\frac{1}{4}\left[2 \Delta(c+1) x^{4}+4 \bar{\sigma}(c+1) x^{3}-\left(4 \Delta c+\bar{\sigma}^{2}\right) x^{2}\right. \\
& \left.\left.+4 \bar{\sigma}(1-c) x+2 \Delta c-2 \Delta+\bar{\sigma}^{2}\right]\right\}
\end{aligned}
$$

By differentiation it may be shown that Eq. (31) is equivalent to the equation $\frac{\partial I}{\partial x}=0$. It is easy to show that when $u^{*}>u_{0}^{*}$ there exists only one solution for the phase interface circle of radius $b$. Dependence of the total energy (32) on $b$ is given in Fig. 4, where only one minimum exists if $u^{*}>u_{0}^{*}$. 


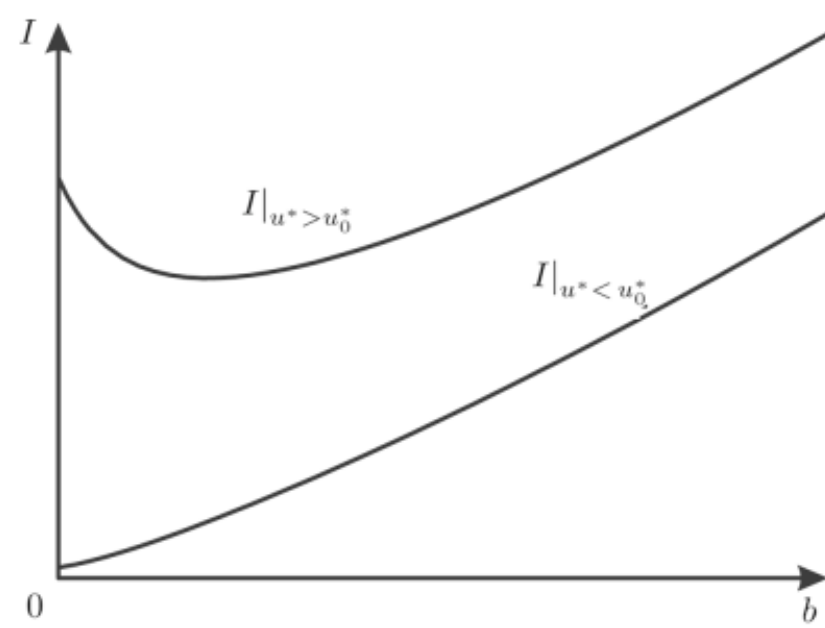

Fig. 4 Dependence of the total energy on the interface radius $b$ for different values of $u^{*}$.

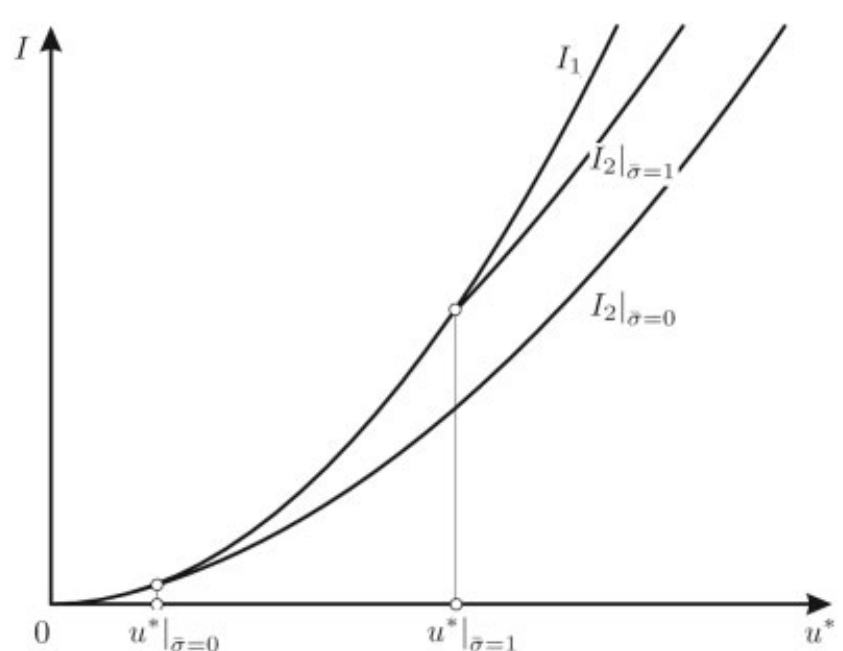

Fig. 5 Total energies for two-phase solution $I_{2}$ and for one-phase solution $I_{1}$.

The solution corresponding to the two-phase equilibrium state is energetically preferable. Let us calculate the value of the functional $I$ on the two-phase solution as well as on the corresponding one-phase solution for different values of $\bar{\sigma}$. Then the total potential energy $I_{2}$ corresponding to the two-phase equilibrium state is always lower than $I_{1}$ corresponding to the one-phase state, see Fig. 5.

\section{Conclusions}

The non-linear quasi-static boundary-value problem of PTs in shells proposed in [1] has been extended by taking into account the elastic strain energy density of the interface as well as the resultant forces and couples acting along the curvilinear interface itself. From the variational principle of total potential energy we have derived the extended static natural continuity conditions to be satisfied at the interface. The conditions allow one to establish position of the interface curve in the thermodynamic equilibrium state of PT.

We have applied the general dynamically and kinematically exact shell model. Therefore, all simplified versions of shell theory with PT can be derived by an appropriate specialization of our results.

The variational principle (7) and the static natural continuity conditions (20) and (22) may be useful in determining the equilibrium position of a movable singular curve in the shell describing other types of singular phenomena. For example, by analogy to the theory of dislocations in 3D bodies [34] the approach developed here may be applied to determination of equilibrium of thin films with dislocations and/or of thin-walled structures with singular curvilinear defects of any nature.

Acknowledgements The first and third author were partially supported by the Polish Committee for Scientific Research under grant No 5 T07A 008 25, while the second author by the Russian Foundation of Basic Research under grant No 04-01-00431 and the Russian Science Support Foundation.

\section{References}

[1] V.A. Eremeyev and W. Pietraszkiewicz, J. Elast. 74, 67-86 (2004).

[2] A. Libai and J. G. Simmonds, The Nonlinear Theory of Elastic Shells, 2nd ed. (University Press, Cambridge, 1998).

[3] J. Chróścielewski, J. Makowski, and W. Pietraszkiewicz, Statics and Dynamics of Multifold Shells: Nonlinear Theory and Finite Element Method (in Polish) (Wydawnictwo IPPT PAN, Warszawa, 2004).

[4] W. Pietraszkiewicz, J. Chróścielewski, and J. Makowski, in: Shell Structures: Theory and Applications, edited by W. Pietraszkiewicz and C. Szymczak (Taylor \& Francis, London, 2005), pp. 163-167.

[5] E. Cosserat and F. Cosserat, Théorie des corps deformables (Herman et Flis, Paris, 1909). English translation: NASA TT F-11, 561 (NASA, Washington D.C., 1968).

[6] P.A. Zhilin, Int. J. Solids Struct. 12, 635-648 (1976).

[7] P.A. Zhilin, Dinamika i Prochnost' Mashin, Trudy LPI 386, 29-46 (1982). 
[8] H. Altenbach and P.A. Zhilin, Adv. in Mech. 11, 107-148 (1988).

[9] J.W. Gibbs, Transactions of the Connecticut Academy of Sciences 3 (1875/1878) 108-248, (1875) 343-354. Reprinted in: The Collected Works of J. Willard Gibs (Longmans, Green \& Co., New York et al., 1928), pp. 55-353.

[10] M. Grinfeld, Thermodynamics Methods in the Theory of Heterogeneous Systems (Longman, Harlow, 1991).

[11] K. Bhattacharya and R.V. Kohn, Arch. Rational Mech. Anal. 139, 99-180 (1997).

[12] A. Mielke, F. Theil, and V. I. Levitas, Arch. Rational Mech. Anal. 162, 137-177 (2002).

[13] Ya.S. Podstrigach and Yu.Z. Povstenko, Introduction to Mechanics of Surface Phenomena in Deformable Solids (in Russian) (Naukova Dumka, Kiev, 1985).

[14] Yu.Z. Povstenko, J. Colloid \& Interface Sci. 144, 497-506 (1991).

[15] L. Wang, Phys. Rev. E 55, 1732-1738 (1997).

[16] A.A. Atai and D. J. Steigmann, Int. J. Solids Struct. 35, 1915-1952 (1998).

[17] A.A. Boulbitch, Arch. Appl. Mech. 69, 83-93 (1999).

[18] K. Bhattacharya and R. D. James, J. Mech. Phys. Sol. 36, 531-576 (1999).

[19] Y.C. Shu, Arch. Rational Mech. Anal. 153, 39-90 (2000).

[20] R. D. James and R. Rizzoni, J. Elast. 59, 399-436 (2000).

[21] A. Roytburd and J. Slutsker, Acta Mater. 50, 1809-1824 (2002).

[22] M.E. Gurtin and M.E. Jabbour, Arch. Rational Mech. Anal. 163, 171-208 (2002).

[23] M. B. Rubin and Y. Benveniste, J. Mech. Phys. Sol. 52, 1023-1052 (2004).

[24] V. Konopińska and W. Pietraszkiewicz, in: Shell Structures: Theory and Applications, edited by W. Pietraszkiewicz and C. Szymczak (Taylor \& Francis, London, 2005), pp. 135-138.

[25] V. Konopińska and W. Pietraszkiewicz, Int. J. Solids Struct. 44, 352-369 (2007).

[26] W. M. Smoleński, Comp. Methods Appl. Mech. Eng. 178, 89-113 (1999).

[27] M.E. Gurtin and A. I. Murdoch, Arch. Rational Mech. Anal. 57, 291-323 (1975).

[28] A. I. Murdoch, Glasgow Math. J. 32, 299-307 (1990).

[29] V.A. Eremeyev and W. Pietraszkiewicz, in: Mechanics of the 21 st Century, edited by W. Gutkowski and T. Kowalewski (SpringerVerlag, Dordrecht, 2005), CD-ROM, Paper No SM19L_10287.

[30] P. Cermelli, M.E. Gurtin, and M. M. Shvartsman, Meccanica 33, 587-599 (1998).

[31] C. Truesdell, A First Course in Rational Continuum Mechanics (Academic Press, New York, 1977).

[32] R. Finn, Equilibrium Capillary Surfaces (Springer-Verlag, New-York et al., 1986).

[33] A. I. Rusanov, Surf. Sci. Rep. 58, 111-239 (2005).

[34] J.P. Hirth and J. Lothe, Theory of Dislocations (McGraw-Hill, New York, 1968). 